\title{
The Neural and Cognitive Time Course of Theory of Mind
}

\author{
Joseph P. McCleery, ${ }^{1}$ Andrew D. R. Surtees, ${ }^{1}$ Katharine A. Graham, ${ }^{1}$ John E. Richards, ${ }^{2}$ and Ian A. Apperly ${ }^{1}$ \\ ${ }^{1}$ School of Psychology, University of Birmingham, Edgbaston, Birmingham B15 2TT, West Midlands, United Kingdom, and ${ }^{2}$ Department of Psychology, \\ University of South Carolina, Columbia, South Carolina 29208
}

Neuroimaging and neuropsychological studies implicate both frontal and temporoparietal cortices when humans reason about the mental states of others. Here, we report an event-related potentials study of the time course of one such "theory of mind" ability: visual perspective taking. The findings suggest that posterior cortex, perhaps the temporoparietal cortex, calculates and represents the perspective of self versus other, and then, later, the right frontal cortex resolves conflict between perspectives during response selection.

\section{Introduction}

"Theory of mind" (ToM) judgments about what others see, know, or think require a range of functional processes and recruit a reliable set of brain regions (Frith and Frith, 2003; Carrington and Bailey, 2009). It is commonly supposed that this constitutes a ToM network, but we currently lack evidence about the timing of these functional and neural processes in real time. Interpretations of the first functional magnetic resonance imaging (fMRI) studies argued that $\mathrm{mPFC}$ was of primary importance for calculating and representing someone else's perspective (Gallagher et al., 2000; Frith and Frith, 2003), while later studies emphasized the importance of temporoparietal junction (TPJ) (Saxe and Kanwisher, 2003). The likely involvement of both of these regions converges with evidence from temporally sensitive, event-related potential (ERP) recordings. Several studies have shown that ToM judgments elicit a late slow activity over frontal (Sabbagh and Taylor, 2000; Liu et al., 2009) and right posterior areas of scalp (Liu et al., 2009), beginning 500-1000 ms after the experimental stimulus. However, since these frontal and posterior effects were observed in different ERP studies, it is not possible to make inferences about the relative involvement of these brain regions across time.

Recent fMRI studies have led to more nuanced claims about potential roles for both the TPJ and PFC (Perner et al., 2006; Schmitz and Johnson, 2007). Nonetheless, it remains unclear how these regions interact with each other or with functional and neural processes for executive function. For example, neuroimaging evidence suggests that lateral prefrontal cortex (lPFC) - a region associated with inhibitory control (Vendrellet al., 1995; Ridderinkhof et al., 2004) — is recruited when participants judge perspectives that differ from their own (Vogeley et al., 2001), and

\footnotetext{
Received March 18, 2011; revised July 7, 2011; accepted July 19, 2011.

Author contributions: J.P.M., A.D.R.S., and I.A.A. designed research; J.P.M., A.D.R.S., and K.A.G. performed research; J.P.M., A.D.R.S., K.A.G., J.E.R., and I.A.A. analyzed data; J.P.M., A.D.R.S., J.E.R., and I.A.A. wrote the paper.

We thank Dr. Sandra Utz and Consuelo del Grande for assistance with data collection, and Emma Cross for assistance with data collection and analysis.

The authors declare no competing financial interests.

Correspondence should be addressed to lan A. Apperly, School of Psychology, University of Birmingham, Edgbaston, Birmingham B15 2TT, West Midlands, United Kingdom. E-mail: i.a.apperly@bham.ac.uk.

DOI:10.1523/JNEUROSCI.1392-11.2011

Copyright $\odot 2011$ the authors $\quad 0270-6474 / 11 / 3112849-06 \$ 15.00 / 0$
}

neuropsychological evidence suggests that strong egocentric interference may result from injury to right PPC (Samson et al., 2005). However, it remains to be discovered whether IPFC is supporting calculation of another's perspective, or the process of selecting between self and other perspectives to make a judgment. Evidence about the timing of activity associated with these different neural regions would be critically informative about the potential interrelationships of ToM processing and executive functions.

Here, we combine ERP recordings with a recently developed task in which participants make rapid ToM judgments about the visual perspectives of self and other, which could be mutually consistent or inconsistent (Samson et al., 2010). Findings from this paradigm suggest a temporal and functional separation between an earlier process of perspective calculation that does not require inhibitory control and a later process of perspective selection that does require inhibitory control (Qureshi et al., 2010). We expected that perspective calculation would yield an ERP component that discriminated Self trials from Other trials, the topology of which would indicate whether frontal or temporoparietal systems were recruited. We further expected that perspective selection would yield an ERP component that discriminated between trials where perspectives were consistent versus inconsistent, and we predicted this to occur over lateral prefrontal regions of scalp, reflecting recruitment of neural systems for inhibitory control.

\section{Materials and Methods}

\section{Overview}

On each trial, participants viewed a picture of a room with discs on the wall, and an avatar whose position in the room meant he saw fewer discs than the participant (perspectives Inconsistent) or all of the discs that the participant could see (perspectives Consistent). On Other-perspective trials, the picture was preceded by a sentence describing the avatar's perspective (e.g., "He sees three"), and participants judged whether the avatar's perspective corresponded to what was said in the sentence (Samson et al., 2010) (Fig. 1). On Self-perspective trials, the picture was preceded by a sentence such as "You see three," and participants judged the number of discs that they themselves could see. Therefore, this paradigm orthogonally varied Perspective judgments (Self vs Other) with the Consistency between self and other perspectives. Response times and scalp potentials were recorded from the onset of the test picture (Fig. 1). 


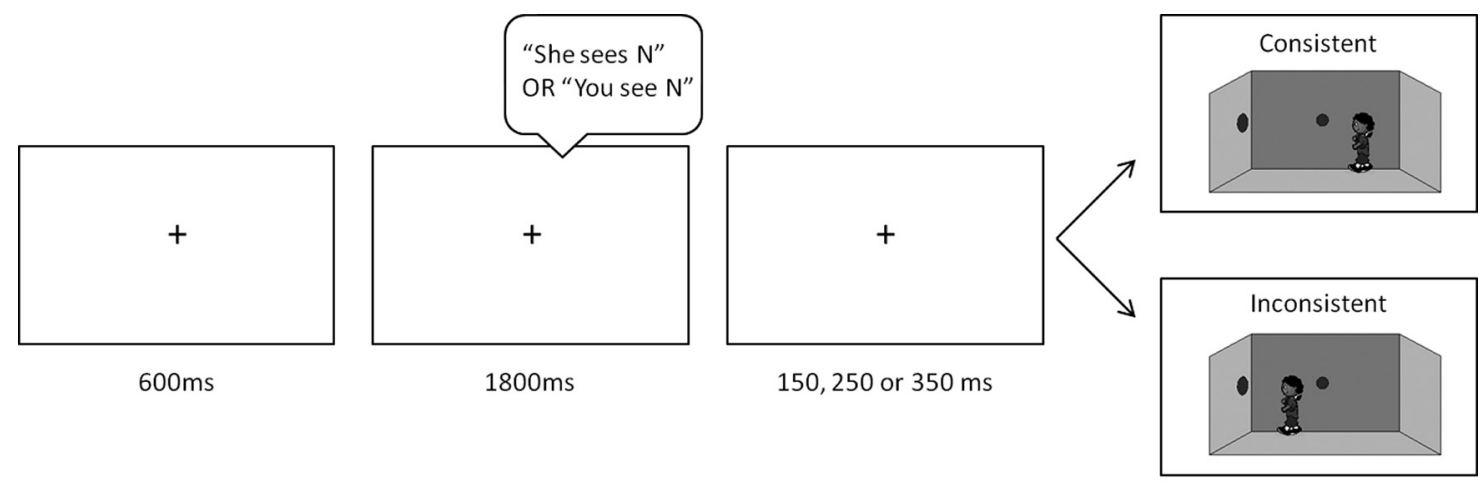

Until response or $1000 \mathrm{~ms}$

Figure 1. Timeline of a trial. On each trial, participants viewed a picture of a room with discs on the wall. The picture was preceded by a spoken sentence that provided a cue to perspective (Self, Other/Avatar) and a numerical value $(1,2,3)$. Participants judged whether the numerical value was an accurate description of the perspective indicated (Self perspective, Other/Avatar perspective). Behavioral response times and scalp potentials were recorded from the onset of the test picture.

\section{Participants}

Participants were 17 undergraduate students (11 female, 6 male; 2 left handed, 15 right handed) at the University of Birmingham. These participants had a mean age of 21.5 years (range: $18-38$ years). Data from five additional participants were excluded from analysis when it was determined that they produced $<30$ artifact-free trials in one or more of the four conditions.

\section{Design and procedure}

Instructions included a detailed description of the procedure and an instruction to respond as quickly and accurately as possible. Practice trials were completed with feedback until the participant successfully answered at least one question for each of the four conditions (SelfConsistent, Self-Inconsistent, Other-Consistent, Other-Inconsistent).

On each trial, participants viewed three fixation stimuli that were presented without a perceptible interval. The first fixation cross $(600 \mathrm{~ms})$ was followed by a second fixation cross (1800 ms) accompanied by an auditory stimulus, followed by a third fixation cross for an additional variable interval of 150-350 ms. The auditory stimulus was either "He sees $N$ " (for Other trials) or "You see $N$ " (for Self trials), where $N$ ranged from 1 to 3 . The fixation stimuli were followed by a test picture, depicting an avatar in a room with between 1 and 3 discs on the wall, so that the number of discs was within the range that could be enumerated quickly and accurately via subitization (Trick and Pylyshyn, 1994). On half of the trials, the auditory stimulus matched the picture, and on half it did not. Participants pressed one of two response pad keys to indicate whether or not the auditory stimulus correctly described the picture (Correct $=$ Key 1 , Incorrect $=$ Key 2). Response time was measured from the onset of the test picture. The picture was displayed on the screen until a response occurred or for a maximum of $1000 \mathrm{~ms}$. Following practice trials, participants completed 768 test trials, equally divided between the four conditions (Self-Consistent, Self-Inconsistent, Other-Consistent, Other-Inconsistent). Self and Other trials were pseudorandomly mixed within each block of trials, such that no block contained more than three trials in a row without a change in consistency, perspective, response button, and direction of avatar. The experiment was presented using E-prime 2.0 (Psychology Software Tools).

\section{EEG recording}

EEG was recorded continuously using a 128 channel Hydrocel Geodesic Sensor Net (HCGSN, Electrical Geodesics) (Tucker, 1993), referenced to a single vertex electrode, $\mathrm{Cz}$ (sample rate $=500 \mathrm{~Hz}$; on-line high-pass filter $=0.1 \mathrm{~Hz}$ ). Electrode impedances were kept at $<80 \mathrm{~K} \Omega$. Visual test stimuli were presented with dimensions of $20 \mathrm{~cm}$ in width and $11.8 \mathrm{~cm}$ in height on a video monitor at a viewing distance of $50 \mathrm{~cm}$, and therefore subtended a visual angle of $\sim 22.6^{\circ}$ horizontal by $\sim 13.5^{\circ}$ vertical.

EEG recordings were processed off-line using NetStation 4.2 software (Electrical Geodesics). The data were filtered (bandpass filter $=0.3-40$ $\mathrm{Hz}$, finite impulse response) and segmented to epochs beginning $100 \mathrm{~ms}$ before and continuing $850 \mathrm{~ms}$ after the presentation of the visual stimuli.
Data were processed using an artifact detection tool that marked channels bad if the recording was poor for $>20 \%$ of the time (threshold maximum - minimum, $>100.00$ ), if eye blinks occurred (threshold maximum - minimum, $>100.00$ ) and/or if eye movements occurred (threshold maximum - minimum, >55.00). Segments were marked bad if they contained $>10$ bad channels, eye blinks, and/or eye movements. Bad channels in the data were replaced using a spherical spline interpolation algorithm (Srinivasan et al., 1996). Each trial was then examined individually to remove any trials with remaining eye-blink or eyemovement artifacts from further analysis. The data were then averaged for each participant, re-referenced to an average reference, and baseline corrected to a $100 \mathrm{~ms}$ prestimulus interval.

\section{Source analysis: estimation of equivalent current dipoles and current densities}

Average MRI. An average head was used for the analyses. The average came from 87 participants ranging in age from 20 to 24 years. The MRI data were collected on a Siemens Medical Systems 3T Trio with an overall duration of approximately $15 \mathrm{~min}$. A 3D, T1-weighted, MPRAGE radio frequency-spoiled rapid flash scan in the sagittal plane and a T2weighted, multislice, axial 3D, dual Fast Turbo spin-echo scan in the sagittal plane were used. The T1 scans had $1 \mathrm{~mm}^{3}$ resolution and sufficient FOV to cover from the top of the head down to the neck.

The T1-weighted MRI images were averaged with the procedures described by Sanchez et al. (2011) (compare with Avants et al., 2008). The procedure involved an iterative process where a tentative MRI average was made. The original MRI volumes were then registered to this tentative volume and transformed in size and orientation with nonlinear registration [using ANTS (Advanced Normalization Tools)] (Avants et al., 2008) into the tentative average space. A new average was constructed from the transformed MRI files. This new average then became the next reference template for the registrations. This procedure results in an average MRI template that is approximately the same volume as the average volume size from the individual MRIs. The average MRI was rotated so that its orientation was approximate to that of the ICBM-152 template, which is oriented to MNI space (Collins et al., 1994, MNI-305 defined; Mazziotta et al., 2001, ICBM-152 defined; Joshi et al., 2004).

Electrodes. Electrodes for the average head were obtained from an average electrode map. This came from a database of 93 individuals whose "geodesic sensor net" (GSN, EGI) (Tucker, 1993) electrode positions were measured with a Geodesic Photogrammetry System (EGI) (Russell et al., 2005). The participants also had a T1-weighted structural MRI. The electrodes were mapped into the average MRI space by registering the $\mathrm{T} 1$ weight of each individual to the average MRI with a 12 degrees of freedom affine registration (Linear Image Registration Tool, Oxford Centre for Functional MRI of the Brain) (Jenkinson and Smith, 2001), transforming the electrodes into the average space, and averaging the electrode positions. This resulted in 
electrode positions located on the average MRI derived from the individual participants.

Processing in CURRY. The average MRI with the electrode positions was used as the head model in Curry 6.0.16 (Neuroscan Compumedics), which allows for both dipole and S-LORETA-based source estimations. The volume conductor model was a three-compartment realistic boundary element model (Fuchs et al., 1998), modeling the surface of the skin, the outside/skull, and the inside of the skull (liquor) with a total of 6361 triangle nodes. The conductivities of the skin, skull, and liquor were set to $0.33,0.042$, and 0.33 , respectively. The average head had segmented gray and white matter. Source locations for the current dipoles and current densities were constrained to $3 \mathrm{~mm}$ tetrahedra volumes of the gray matter. Talairach locations were obtained from CURRY, which translates the head size and positions of the average MRI into the head size and positions for the Talairach stereotaxic atlas (Talairach and Tournoux, 1988).

\section{Results}

\section{Behavioral results}

Response times

An ANOVA with Perspective and Consistency as within-subjects factors revealed a significant effect of Perspective $\left(F_{(1,16)}=\right.$ 31.753; $p<0.001, \eta p^{2}=0.665$; Self $=526.53 \mathrm{~ms}$; Other $=569.07$ $\mathrm{ms})$, a significant effect of Consistency $\left(F_{(1,16)}=210.534 ; p<\right.$ $0.001, \eta p^{2}=0.929$; Consistent $=518.02 \mathrm{~ms}$; Inconsistent $=$ $577.58 \mathrm{~ms}$ ), and an interaction between Perspective and Consistency $\left(F_{(1,16)}=7.337 ; p=0.015, \eta p^{2}=0.314\right)$. The effect of Consistency was greatest for Other perspective-taking $\left(t_{(16)}=\right.$ 13.444, $p<0.001$; Consistent $=533.11 \mathrm{~ms}$; Inconsistent $=$ $605.03 \mathrm{~ms}$ ), but also significant for Self Perspective-taking $\left(t_{(16)}=6.904, p<0.001\right.$; Consistent $=502.93 \mathrm{~ms}$; Inconsistent $=$ $550.13 \mathrm{~ms})$.

\section{Errors}

A similar ANOVA on errors revealed no significant effect of Perspective $\left(F_{(1,16)}=0.001, p=0.974, \eta p^{2}<0.001\right)$. There was a significant effect of Consistency $\left(F_{(1,16)}=30.700, p<0.001\right.$, $\eta p^{2}=0.657$; Consistent $=2.45 \%$; Inconsistent $\left.=9.84 \%\right)$. There was not a significant interaction between Perspective and Consistency $\left(F_{(1,16)}=2.561, p=0.129, \eta p^{2}=0.138\right)$.

\section{ERP results}

\section{ERP components}

Five ERP components were identified via the piloting of an initial eight adult participants whose data are not included in the current report. These components were early components peaking at $\sim 200$ ms over the left and right lateral frontal (FL190; negativegoing) and central occipital cortices (P200; positive-going), respectively; a middle-latency component peaking between $\sim 400$ and $500 \mathrm{~ms}$ recorded from electrodes over the left and right temporoparietal cortices (TP450; positive-going); and a late frontal slow-wave (LFSW) component with a mean amplitude difference between 600 and $800 \mathrm{~ms}$ recorded over the right frontal cortex. These same components were also clearly observed in the 17 participants in the current experiment. Electrodes used to measure each component were determined by examination of both the grand average and individual subject data of the pilot participants, and then confirmation of these as appropriate electrodes for the final 17 participants whose data are reported here. The electrodes selected for pilot data analysis were also deemed to be the most appropriate for the final 17 participants, and are as follows: left frontal: $25,26,27,32,33$; right frontal: 1, 2, 8, 122, 123; occipital: $69,70,73,74,75,81,82,83,88$, 89; left temporoparietal: $30,36,37,41,42,46,47,52,53$; right temporoparietal: $86,87,92,93,98,102,103,104,105$. Peak amplitudes, latencies to peak amplitudes, and mean amplitudes (i.e., LFSW) were measured for each individual electrode in the relevant montages and then averaged within relevant regions for each participant. Time windows for each component were as follows: F190: 160-240 ms; P200: 165-230 ms; TP450: 325-525; LFSW: 600-800 ms. Only trials in which participants correctly responded "yes" were included in the analyses.

Three-factor ANOVAs including Perspective (self, other), Consistency (consistent, inconsistent), and Hemisphere (left, right) as within-subjects factors were conducted on the latency and amplitude data for the temporoparietal and lateral frontal components. Two-factor ANOVAs including Perspective and Consistency as within-subjects factors were conducted on the latency and amplitude data for the central occipital component.

\section{ERP component effects}

The latencies of the early frontal cortex (FL190) component exhibited a Perspective-by-Hemisphere interaction $\left(F_{(1,16)}=\right.$ 39.425, $p<0.001, \eta p^{2}>0.99$ ), with longer latency for Self than Other over the right hemisphere, and the reverse pattern over left hemisphere. The amplitudes of this same component also exhibited a Perspective-by-Consistency-by-Hemisphere interaction $\left(F_{(1,16)}=9.969, p=0.006, \eta p^{2}=0.842\right)$, whereby Self judgments on Inconsistent trials elicited a larger amplitude response over the right hemisphere only. Simultaneous to this, the early central occipital component (P200) also exhibited a Perspective-byConsistency interaction $\left(F_{(1,16)}=4.53, p=0.049, \eta p^{2}=0.516\right)$, whereby Self judgments on Inconsistent trials elicited a larger amplitude response.

The latencies of the middle-latency temporoparietal component (TP450) exhibited a main effect of Perspective, whereby latencies for Other perspective judgments were longer than for Self-perspective judgments $\left(F_{(1,16)}=53.315, p<0.001, \eta p^{2}=\right.$ $1.0)$. However, this main effect was moderated by a Perspectiveby-Consistency interaction $\left(F_{(1,16)}=10.619, p=0.005, \eta p^{2}=\right.$ 0.864 ), whereby latencies for Other perspective judgments were longer than for Self-perspective judgments, with Other Inconsistent trials eliciting the longest latency responses (Self-Consistent $=417 \mathrm{~ms}, \mathrm{SE}=8.6$; Self-Inconsistent $=409 \mathrm{~ms}, \mathrm{SE}=9.3$; Other-Consistent $=444 \mathrm{~ms}, \mathrm{SE}=9.0$, Other-Inconsistent $=476$ $\mathrm{ms}, \mathrm{SE}=7.4)$. The amplitudes of the TP450 exhibited a main effect of Consistency $\left(F_{(1,16)}=6.628, p=0.020, \eta p^{2}=0.293\right)$ and a main effect of Hemisphere $\left(F_{(1,16)}=9.903, p=0.006\right.$, $\left.\eta p^{2}=0.382\right)$, whereby amplitudes were larger for Consistent compared with Inconsistent trials, and larger over the right hemisphere compared with the left. TP450 amplitudes also exhibited a Perspective-by-Hemisphere interaction $\left(F_{(1,16)}=6.026, p=\right.$ $\left.0.026, \eta p^{2}=0.274\right)$, and a trend for a Consistency-by-Hemisphere interaction $\left(F_{(1,16)}=4.217, p=0.057, \eta p^{2}=0.209\right)$, where in both cases the pattern was for condition differences seen in Figure 2 to be larger in the left hemisphere compared with the right hemisphere. Most notably, the Self Inconsistent condition exhibited right lateralized activity, whereas all other conditions were distributed more bilaterally (Fig. 2).

The LFSW (600-800 ms) exhibited a Consistency-by-Hemisphere interaction $\left(F_{(1,16)}=39.425, p<0.001, \eta p^{2}>0.99\right)$, whereby mean amplitudes differed for Consistent versus Inconsistent over the right hemisphere only (Fig. 3).

\section{Source estimates}

Although the TP450 effect was limited to a particular region of the scalp, it occurred simultaneously with activity of a larger posterior component. The larger component during this time window 
likely reflects occipital, temporal, and parietal activity that is shared across our conditions. However, the existence of this broad component simultaneously with the TP450 component of interest complicates unconstrained source analysis procedures, such as s-LORETA and unconstrained current source dipole analyses, particularly when deep sources are implicated. This complication was, in fact, observed in S-LORETA analyses. These analyses, which followed the constrained dipole analyses described here, confirmed that the source solutions using this unconstrained method were dominated by deep sources in the occipital, temporal, and parietal lobes in all conditions. Therefore, given our strong hypothesis that the differences observed in the TP450 component reflect differences in the posterior ToM region, the temporoparietal junction, we conducted confirmatory equivalent dipole analyses with dipoles located in the gray matter of the temporoparietal junction during the time of the TP450 component (fixed location, rotating amplitude vector) (Fig. 4). A model with a unilateral dipole in the right TPJ (rTPJ) (Talairach coordinates: 60, -40 , 20) accounted for 63,60 , or $70 \%$ of variance for the Self-Consistent, Other-Consistent, and Other-Inconsistent conditions, respectively; adding a second dipole in the left TPJ (lTPJ) [Talairach coordinates: $+/-60,-40,20$ (e.g., bilateral TPJ dipoles)] added a significant amount of explained variance $(27,27,21 \%$, respectively). For the SelfInconsistent scalp ERP, the unilateral rTPJ model accounted for $76 \%$ of the variance, and a bilateral model added only $13 \%$ explained variance. For all of these models, additional dipoles located in the medial prefrontal lobes did not add significant additional explained variance $(<4 \%)$. This confirmatory source analysis for the TP450 component is consistent with a model of bilateral temporoparietal junction activity for Self-Consistent, Other-Consistent, and Other-Inconsistent; predominantly unilateral right temporoparietal junction involvement for Self-Inconsistent; and primarily posterior and lateral sources of brain activity underlying the TP450 results.

To estimate sources for neural activity during the time of the late frontal slow-wave component, we used Curry's S-LORETA with Lp Norm equal to 2, with sources constrained to the gray matter. Three sources were observed for the 600-800 ms time window of the late frontal slow wave component difference. The first of these was a source in the right anterior portion of the inferior frontal gyrus and middle frontal gyrus (Talairach coordinates: $37,55,-5)$ that differed for the Consistent versus Inconsistent trials for both the Self and Other conditions. The second was a medial orbital frontal source that was present in all four conditions (Talairach coordinates: $-1,37,-21$ ). Finally, a left temporal pole source was observed in all conditions except Self Inconsistent (Talairach coordinates: $-24,8,-60$ ) (Fig. 5).

\section{Discussion}

The current study combined a ToM task that required rapid processing of information about perspectives together with EEG re-

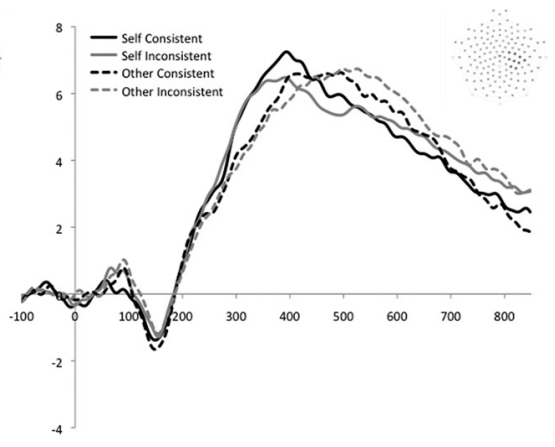

Figure 2. Left and right hemisphere temporoparietal waveforms. The TP450 component latencies exhibited a Perspective (Self, with Inconsistent trials, and larger over right hemisphere compared with left. Amplitude effects tended to be larger in the left hemisphere compared with the right hemisphere.
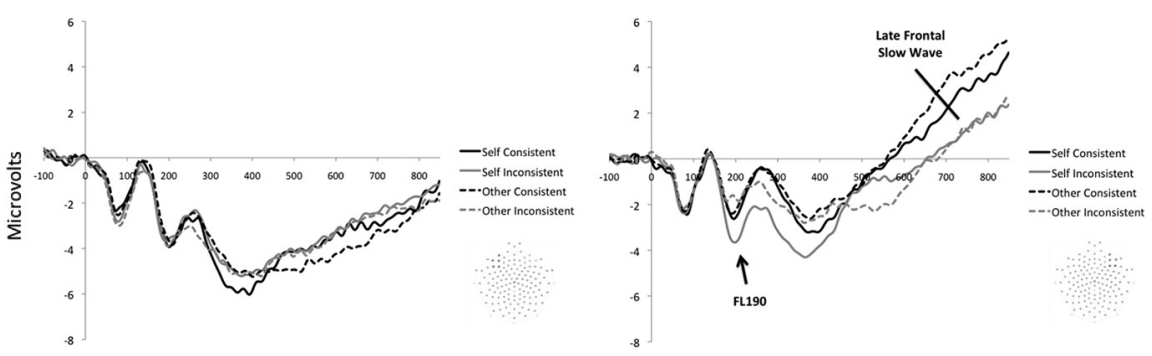

$\cdot 8$

Figure 3. Left and right hemisphere lateral frontal waveforms. The late frontal slow wave component exhibited a Consistencyby-Hemisphere interaction, whereby mean amplitudes differed for Consistent versus Inconsistent over the right hemisphere only. The amplitudes of the early FL190 component exhibited a Perspective-by-Consistency-by-Hemisphere interaction, whereby Self judgments on Inconsistent trials elicited a larger amplitude response over the right hemisphere only.

cording. It is commonly supposed that ToM judgments require multiple functional and neural processes, perhaps including some that are truly specific to ToM, and most likely also including generic processes for executive control (Van Overwalle, 2009). However, most studies of ToM have been unable to distinguish between these component processes and study their relative time course. The ToM task in the current study is unusually well suited to this purpose because existing behavioral data indicate a distinction between an initial process of perspective calculation followed by a process of selecting the appropriate (Self or Other) perspective to respond on a given trial. Perspective calculation is not disrupted when participants perform a dual task that taxes inhibitory control (suggesting that it does not require general cognitive processes for inhibition), whereas the same dual task does disrupt perspective selection (Qureshi et al., 2010). By combining this task with EEG recording that allowed neural responses to the task to be monitored with high temporal precision, we found several distinct neural processes, indexed as ERP components. We discuss these components in turn.

Two early components were observed over the lateral frontal and central occipital cortices peaking at 190 and $200 \mathrm{~ms}$, respectively, after the presentation of the test picture. Both components showed larger amplitudes for Self-Inconsistent trials in comparison with the other three trial types. A distinctive characteristic of this condition is that it is the only trial type in which participants must attend to discs appearing on the wall behind the character. In contrast, on both Self-Consistent and Other-Consistent trials, the discs all appear in front of the character, whereas on OtherInconsistent trials, participants must attend to discs appearing in 

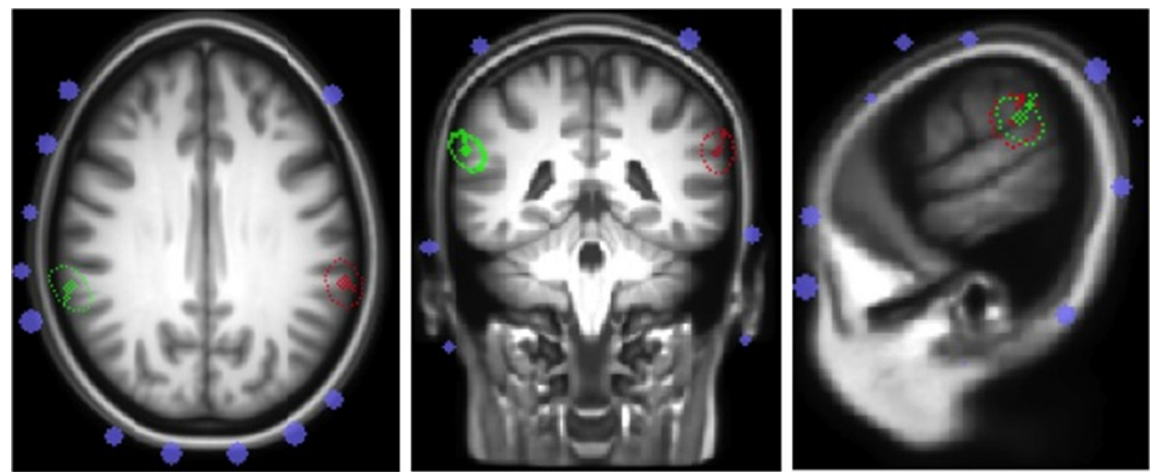

Figure 4. Locations of fixed rotating current source dipoles for TP450 component analysis. Fixed, rotating dipoles were placed in the gray matter of the TPJ. A model with a dipole in the rTPJ accounted for 63,60 , or $70 \%$ of variance for the Self-Consistent, Other-Consistent, and Other-Inconsistent conditions, respectively; adding a second dipole in the ITPJ added a significant amount of explained variance $(27,27,21 \%$, respectively). For the Self-Inconsistent condition, the unilateral rTPJ model accounted for $76 \%$ of the variance, and a bilateral model added only $13 \%$ of the explained variance. Additional dipoles in the medial prefrontal lobes did not add significant additional explained variance.

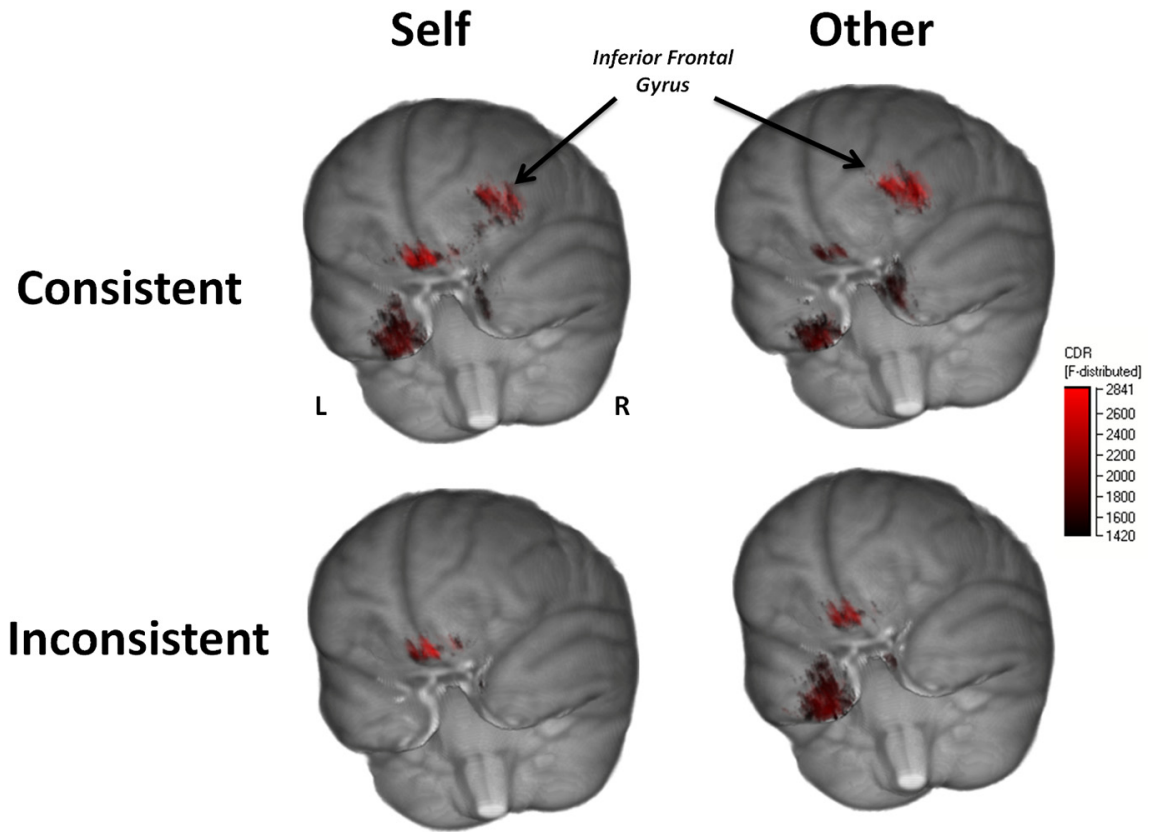

Figure 5. Source estimates for neural activity during the time of the late frontal slow wave. Three sources were observed for the $600-800 \mathrm{~ms}$ time window of the late frontal slow wave component difference. The first of these was a right inferior frontal gyrus source that differed for the Consistent versus Inconsistent trials for both the Self and 0ther conditions. The second was a medial orbital frontal source that was present in all four conditions. Finally, a left temporal pole source was observed in all conditions except Self Inconsistent.

front of the character while ignoring those behind the character. Thus, we believe that both the early frontal and occipital components are likely to be an artifact of strategic visual attention, and so, these effects are unlikely to be informative about ToM processing per se.

The ERP component recorded from leads over the bilateral temporoparietal scalp (TP450) was the first component to reflect the processing costs of calculating the Other's perspective, reflected in longer latencies for Other-perspective judgments that were longest of all when Other was inconsistent with Self. Recall from the introduction that there is much debate about the relative importance of mPFC and TPJ regions in ToM (Frith and Frith, 2003; Saxe and Kanwisher, 2003). The confirmatory equivalent current dipole source analyses are consistent with a primacy for the TP cortex versus the medial frontal cortex in the initial calculation of simple visual perspectives. This interpretation converges with the view that the mPFC is mainly necessary for ToM tasks that entail more complex or uncertain judgments than the simple visual perspectives used in the current study (Aichhorn et al., 2006; Mitchell et al., 2006). It is also notable that all four conditions appeared to have sources in right posterior cortex at the time of the TP450, whereas there was more variability in the recruitment of left posterior cortex. This observation is consistent with neuroimaging evidence for functional differentiation between right and left TPJ, and indeed fits with the view that right $\mathrm{TP}$ cortex may represent any psychological perspective (Self, Other, Consistent, Inconsistent), whereas left TP cortex may be indexing differences in perspective and ownership of perspective (Perner et al., 2006; Aichhorn et al., 2009).

Finally, our findings extend existing studies in identifying the functions served by frontal processes activated during this simple ToM task. First, the late frontal component effect was right lateralized, and was sensitive to inconsistency between perspectives rather than whether participants judged Self or Other perspective. This converges with evidence that right lateral PFC in particular may be involved in managing interference between perspectives (Samson et al., 2005; Saxe et al., 2006). Second, the frontal component was the final component observed, and overlapped in time with participants' responses, suggesting that they must have already completed the calculation of perspectives. Given that behavioral data indicate that this process of perspective selection may be selectively disrupted when participants undertake a dual task that taxes inhibitory control (Qureshi et al., 2010), it seems plausible that differences in this late slow-wave component reflect the differential recruitment of executive processes for inhibitory control, which are strongly associated with right lateral PFC (Vendrell et al., 1995). This component was also associated with sources in medial orbital frontal cortex and left temporal pole, regions that have been implicated in some fMRI studies of ToM (Frith and Frith, 2003; Van Overwalle, 2009). However, unlike the source in right lateral PFC, these sources were not reliably sensitive to perspective consistency or Self versus Other perspective, and so the variables of our perspective-taking task cast no new light on the function of these regions.

In sum, our findings reveal both functional and temporal differentiation in the neural processes recruited for a type of ToM ability, visual perspective taking. Following initial visual analysis of the stimuli, posterior regions of cortex, perhaps the left and right temporoparietal cortex, are involved in calculating and rep- 
resenting Self versus Other perspectives. A later right frontal component reflects the exercise of executive functions for cognitive management of perspective selection. These findings reveal the stages of processing involved in ToM judgments for visual perspective taking, and also open the way for asking how ToM processing develops, as well as how it may be impaired in disorders of social cognitive functioning (Senju et al., 2009).

\section{References}

Aichhorn M, Perner J, Kronbichler M, Staffen W, Ladurner G (2006) Do visual perspective tasks need theory of mind? Neuroimage 30:1059-1068.

Aichhorn M, Perner J, Weiss B, Kronbichler M, Staffen W, Ladurner G (2009) Temporo-parietal junction activity in theory-of-mind tasks: falseness, beliefs, or attention. J Cogn Neurosci 21:1179-1192.

Avants BB, Epstein CL, Grossman M, Gee JC (2008) Symetricdiffeomorphic image registration with cross-correlation: evaluating automated labeling of elderly and neurodegenerative brain. Med Image Anal 12:26-41.

Carrington SJ, Bailey AJ (2009) Are there theory of mind regions in the brain? A review of the neuroimaging literature. Hum Brain Mapp 30:2313-2335.

Collins DL, Neelin P, Peters TM, Evans AC (1994) Automatic 3D intersubject registration of MR volumetric data in standardized Talairach space. J Comput Assist Tomogr 18:192-205.

Frith U, Frith CD (2003) Development and neurophysiology of mentalizing. Philos Trans R Soc Lond B Biol Sci 358:459-473.

Fuchs M, Drenckhahn R, Wischmann HA, Wagner M (1998) An improved boundary element model for realistic volume-conduction modeling. IEEE Trans Biomed Eng 45:980-997.

Gallagher HL, Happé F, Brunswick N, Fletcher PC, Frith U, Frith CD (2000) Reading the mind in cartoons and stories: an fMRI study of "theory of mind" in verbal and nonverbal tasks. Neuropsychologia 38:11-21.

Jenkinson M, Smith S (2001) A global optimisation method for robust affine registration of brain images. Med Image Anal 5:143-156.

Joshi S, Davis B, Jomier M, Gerig G (2004) Unbiased diffeomorphicatlast construction for computational anatomy. Neuroimage 23:S151-S160.

Liu D, Sabbagh MA, Gehring WJ, Wellman HM (2009) Neural correlates of children's theory of mind development. Child Dev 80:318-326.

Liu D, Meltzoff AN, Wellman HM (2009) Neural correlates of belief- and desire reasoning. Child Dev 80:1163-1171.

Mazziotta J, Toga A, Evans A, Fox P, Lancaster J, Zilles K, Simpson G, Woods R, Paus T, Pike B, Holmes C, Collins L, Thompson P, MacDonald D, Iacoboni M, Schormann T, Amunts K, Palomero-Gallagher N, Geyer S, Parsons L, et al (2001) A probabilistic atlas and reference system for the human brain. Philos Trans R Soc Lond B Biol Sci 356:1293-1322.

Mitchell JP, Macrae CN, Banaji MR (2006) Dissociable medial prefrontal contributions to judgments of similar and dissimilar others. Neuron 50:655-663.

Perner J, Aichhorn M, Kronbichler M, Staffen W, Ladurner G (2006)
Thinking of mental and other representations: the roles of the left and right temporo parietal junction. Soc Neurosci 1:245-258.

Qureshi AW, Apperly IA, Samson D (2010) Executive function is necessary for perspective-selection, not level-1 visual perspective-calculation: evidence from a dual-task study of adults. Cognition 117:230-236.

Ridderinkhof KR, Ullsperger M, Crone EA, Nieuwenhuis S (2004) The role of the medial frontal cortex in cognitive control. Science 306:443-447.

Russell GS, Eriksen KJ, Poolman P, Luu P, Tucker DM (2005) Geodesic photogrammetry for localizing sensor positions in dense-array EEG. Clin Neurophysiol 116:1130-1140.

Sabbagh MA, Taylor M (2000) Neural correlates of theory-of-mind reasoning: an event-related potential study. Psychol Sci 11:46-50.

Samson D, Apperly IA, Kathirgamanathan U, Humphreys GW (2005) Seeing it my way: a case of a selective deficit in inhibiting self-perspective. Brain 128:1102-1111.

Samson D, Apperly IA, Braithwaite JJ, Andrews BJ, Bodley Scott SE (2010) Seeing it their way: evidence for rapid and involuntary computation of what others see. J Exp Psychol Hum Percept Perform 36:1255-1266.

Sanchez CE, Richards JE, Almli CR (2011) Neurodevelopmental MRI brain templates for children from 2 weeks to 4 years of age. Dev Psychobiol. Advance online publication. Retrieved June 17, 2011. doi:10.1002/dev.20579.

Saxe R, Kanwisher N (2003) People thinking about thinking people-the role of the temporoparietal junction in "theory of mind." Neuroimage 19:1835-1842.

Saxe R, Schulz LE, Jiang YV (2006) Reading minds versus following rules: dissociating theory of mind and executive control in the brain. Soc Neurosci 1:284-298.

Schmitz TW, Johnson SC (2007) Relevance to self: a brief review and framework of neural systems underlying appraisal. Neurosci Biobehav Rev 31:585-596.

Senju A, Southgate V, White S, Frith U (2009) Mindblind eyes: an absence of spontaneous theory of mind in Asperger syndrome. Science 325:883-885.

Srinivasan R, Nunez PL, Tucker DM, Silberstein RB, Cadusch PJ (1996) Spatial sampling and filtering of EEG with splinelaplacians to estimate cortical potentials. Brain Topogr 8:355-366.

Talairach J, Tournoux P (1988) Co-planar stereotaxic atlas of the human brain. New York: Thieme Medical Publishers.

Trick LM, Pylyshyn ZW (1994) Why are small and large numbers enumerated differently-a limited-capacity preattentive stage in vision. Psychol Rev 101:80-102.

Tucker DM (1993) Spatial sampling of head electrical fields: the geodesic sensor net. Electroencephalogr Clin Neurophysiol 87:154-163.

Van Overwalle F (2009) Social cognition and the brain: a meta-analysis. Hum Brain Mapp 30:829-858.

Vendrell P, Junqué C, Pujol J, Jurado MA, Molet J, Grafman J (1995) The role of prefrontal regions in the Stroop task. Neuropsychologia 33: 341-352.

Vogeley K, Bussfeld P, Newen A, Herrmann S, Happé F, Falkai P, Maier W, Shah NJ, Fink GR, Zilles K (2001) Mind reading: neural mechanisms of theory of mind and self-perspective. Neuroimage 14:170-181. 